\title{
THE ACCURACY OF POPULATION PROJECTIONS
}

Michael A. Stoto

John F. Kennedy School of Government, Harvard University, Cambridge, Massachusetts 02138, USA

RR-84-17

July 1984

Reprinted from Journal of the American Statistical Association. volume 78 (1983)

INTERNATIONAL INSTITUTE FOR APPLIED SYSTEMS ANALYSIS

Laxenburg, Austria 
Research Reports, which record research conducted at IIASA, are independently reviewed before publication. However, the views and opinions they express are not necessarily those of the Institute or the National Member Organizations that support it.

Reprinted with permission from Journal of the American Statistical Association, 78(381):13-20. Copyright (c) 1983 American Statistical Association.

All rights reserved. No part of this publication may be reproduced or transmitted in any form or by any means, electronic or mechanical, including photocopy, recording, or any information storage or retrieval system, without permission in writing from the copyright holder.

Printed by Novographic, Vienna, Austria 


\section{FOREWORD}

Although population studies are key elements of many planning and policy studies, they are inherently inaccurate. This report attempts to measure the inaccuracy through a comparison of past projections with the actual population figures. The aim of this historical study is not to criticize or applaud the quality of previous demographic predictions, but rather to provide confidence intervals for projections made today. This should allow planners to use projections more objectively by providing a range of reasonable possibilities rather than a single estimate.

ANDRZEJ WIERZBICKI

Chairman

System and Decision Sciences Program 



\section{The Accuracy of Population Projections}

Population projections are key elements of many planning and policy studies but are inherently inaccurate. This study of past population projection errors provides a means for constructing confidence intervals for future projections. We first define a statistic to measure projection errors independently of the size of the population and the length of the projection period. A sample of U.S. Census Bureau and United Nations projections indicates that the distributions of components of the error statistic are relatively stable. We then use this information to construct confidence intervals for the total population of the United States through the year 2000. We find that for projections of total population size, simple projection techniques are more accurate than more complex techniques.

KEY WORDS: Population forecasts; Demographic projections; Prediction; Forecasting; Confidence intervals; Modeling.

\section{INTRODUCTION}

Population projections are basic tools both for governmental and for private planners. They ask: how many people will be in an area at a given time in the future? Planners can answer this question in many ways, depending on what assumptions they are willing to make. Keyfitz (1972) offers a catalog of the available techniques.

Planners ask a second question less frequently: within what range can we be confident that the future population will fall? This article answers the second question both theoretically and specifically for the United States in the year 2000.

Population projections are simple mathematical extrapolations of current trends and assumptions about the future. They could be meant to illustrate and compare the results of various policies, or to warn policy makers about the consequences of current trends. But Keyfitz (1972) points out that projections are frequently regarded as predictions, that is, as statements about what is likely to

\footnotetext{
* Michael A. Stoto is Assistant Professor of Public Policy, John F. Kennedy School of Government, Harvard University, Cambridge, MA 02138 . The original calculations for this article were performed while the author was supported by N.S.F. Grant SOC 75-15702 in the Department of Statistics, Harvard University. The writing was supported by the International Institute for Applied Systems Analysis, Laxenburg, Austria. A preliminary version of the article was presented at the annual meeting of the Population Association of America, Philadelphia, Pennsylvania, April 1979. The author would like to thank Volker Abel, Brian Arthur, Jose Gomez de Leon, Herman Leonard, Lincoln Moses, Frederick Mosteller, Donald Pittenger, and Thomas Willemain for stimulating discussions and helpful comments. Special thanks is due to $\mathrm{Na}$ than Keyfitz, who originally suggested the idea of a historical study of the projection accuracy.
}

occur. Users often interpret projections issued by government agencies in this way. Throughout this article we regard all projections as predictions and therefore talk about the accuracy of population projections, interpreted as predictions.

Demographers analyze the accuracy of population projections in two ways. The first is to specify a mathematical model for the growth of population and explore the effects of variation in the inputs. The U.S. Bureau of the Census and the Population Bureau of the United Nations do this informally when they present high, low, and medium series of projections, reflecting different beliefs about the future course of mortality and fertility. Sykes (1969), Lee (1974), Cohen $(1976,1977 a, b)$ and Saboia $(1974,1977)$ do it more formally by developing mathematical models for the variations in vital rates.

This article presents a second, more empirical approach to the same problem. Rather than make assumptions about either the magnitude of possible error in our assumptions or a mechanism for the change in rates, we let the projections speak for themselves. In the past 200 years, competent demographers have made many predictions for target years that have already gone by. These projections embody assumptions about the appropriate projection model, estimates of current values, and assumptions about trends. White (1954), Siegel (1972), and Isserman (1977) have used such data to explore the revealed accuracy of projections.

But as Pittenger (1978) points out, any specific projection is made and evaluated in a limited historical context, but projection errors come about because the world changes in new and unexpected ways. To get results that transcend particular historical situations, we examine a broad temporal and geographical range of projections. For these observed errors to be useful indicators of the potential error of present projections, we must first abstract such obvious determining factors as the length of the projection period and the eventual size of the projected population. To adjust for these factors and obtain a homogeneous measure of error, we define a statistic $\Delta r$ that takes the duration and size factors into account. A statistical study of the distribution of $\Delta r$ then leads us to statements about the probable size of future projection errors.

This article neither criticizes nor applauds the quality of population predictions. Instead it aims to provide confidence intervals for projections made today, assuming

Reprinted from: c) Journal of the American Statistical Association March 1983, Volume 78, Number 381 Applications Section 
that their quality is at least as good as it has been in the past.

\section{DEFINITION OF $\Delta r$}

Constant exponential increase is the simplest model of population growth. According to this theory, if $P_{0}$ is the current population and $r$ is the growth rate, the population $T$ years from now, $P_{T}=P_{0} \exp (r T)$. If the growth rate is not a constant but is instead a function of time, $r(t)$, we write

$$
P_{T}=P_{0} \exp \int_{0}^{T} r(t) d t .
$$

The average growth rate over the projection period is

$$
\bar{r}=\frac{1}{T} \int_{0}^{T} r(t) d t
$$

so even if $r(t)$ is an arbitrary function of time, we can write $P_{T}=P_{0} \exp (\bar{r} T)$. From this it is easy to calculate $\bar{r}=\log _{e}\left(P_{T} / P_{0}\right) / T$.

The average growth rate, $\bar{r}$, is dimensionless and takes the initial and final population size and the duration of the projection period into account in a reasonable way. It sums up in one number the growth of the population over $T$ years. Since $\bar{r}$ relates the true populations at the beginning and end of the projection period, we label it $\bar{r}_{\text {true }}$.

The simplest projection method assumes that the population will grow exponentially with some value $r$. We can describe the entire projection by one number, call it $\bar{r}_{\text {proj }}=r$. For more complex projection methods, we define the average growth rate of the projection in terms of the original estimated population, $\hat{P}_{0}$, and the projected population, $\hat{\boldsymbol{P}}_{T}$, as $\overline{\boldsymbol{r}}_{\text {proj }}=\log _{e}\left(\hat{\boldsymbol{P}}_{T} / \hat{\boldsymbol{P}}_{0}\right) / T$. We use $\hat{\boldsymbol{P}}_{0}$ rather than $\boldsymbol{P}_{\mathbf{0}}$ because the true population at time zero may not be known at the time the projection is made and an estimate used instead.

Finally, we define the error term, $\Delta r$, as the percentage difference of the average growth rates,

$$
\Delta r=100 \times\left(\tilde{r}_{\text {proj }}-\tilde{r}_{\text {true }}\right)=\frac{100}{T} \log _{e}\left(\frac{\hat{P}_{T}}{\hat{P}_{0}} \cdot \frac{P_{0}}{P_{T}}\right) .
$$

The statistic, $\Delta r$, summarizes in one number the error in a population projection. It takes the population size and the projection duration into account. Since we use both $\hat{\boldsymbol{P}}_{0}$ and $\boldsymbol{P}_{0}, \Delta r$ ignores errors caused by a bad estimate of the initial population, or a slight change in the coverage region. In this way, we study the projection itself and its implicit assumptions about growth rates, not errors in the initial population. It does not reflect errors in the age or geographical distribution or the errors in the projection between the base and target years.

\section{ANALYSIS OF U.S. PROJECTIONS}

We first look at some data for the United States. Table 1 presents the $\Delta r$ for projections made by the U.S. Census Bureau $(1946,1953,1958,1962,1966,1971)$ in jump-off
Table 1. $\Delta r$ for U.S. Population Projections

\begin{tabular}{crrrrrr}
\hline \hline & \multicolumn{5}{c}{ Jump-Off Year } \\
\cline { 2 - 7 } Duration & 1945 & 1950 & 1955 & 1960 & 1965 & 1970 \\
\hline 5 & -.86 & -.07 & .02 & .11 & .11 & .32 \\
10 & -.96 & -.18 & .08 & .32 & .32 & \\
15 & -1.05 & -.20 & .26 & .52 & & \\
20 & -1.03 & -.11 & .45 & & & \\
25 & -.97 & -.05 & & & & \\
30 & -.90 & & & 32 & .32 & .32 \\
Average & -.96 & -.12 & .20 & .32 & &
\end{tabular}

years 1945 through 1970 , for target years 1950 through 1975. We present the median projection in all cases.

Treating the 21 values in Table 1 as a random sample, the average error is -.19 and the standard deviation .54 . Since the average $\Delta r$ is negative, the projections have been biased downward, that is they have been undershooting the mark. But a closer look reveals a strong pattern in the data. All of the projections made in 1945 and 1950 were low, and all later projections were high. The average value of $\Delta r$ for each column appears below Table 1. The message seems clear: in 1945 and 1950, the forecasters did not anticipate the baby boom, and after it began they did not realize it would not continue. In the analysis of variance sense the column mean values explain more than 95 percent of the variance in Table 1. ( $F$ $=62.80$ with 5 and 15 degrees of freedom, $p<.001$.) The standard deviation of the residual $\Delta r$, once the means have been removed, is .13, compared to the original .54. In the same sense, neither row nor diagonal means are significant $(F<1)$

Let us identify the average error for each year as the jump-off error. The small residual error partially reflects the fact that the projections were made simultaneously among the experts. Dorn (1950) (see Table 2) presents population projections made during the 1930's and 1940's by Pearl and Reed, Dublin, and the Scripps Institute. Their projections for the United States in 1970 ranged from 145 to 172 million. Since the population turned out to be 205 million, the $\Delta r$ 's for the projections reported by Dorn ranged from -.42 to -1.02 .

To calculate a confidence interval for a future population, we must first estimate the distribution of the error,

Table 2. Population Projections for U.S. 1970 (actual population-204.9 million)

\begin{tabular}{lccrc}
\hline \hline \multicolumn{1}{c}{ Name } & Year & $\begin{array}{c}\text { Projection } \\
\text { (in millions) }\end{array}$ & Base & \multicolumn{1}{c}{$\Delta r$} \\
\hline Pearl-Reed I & $' 10$ & 167.9 & 92.4 & -.33 \\
Pearl-Reed II & $' 30$ & 160.4 & 123.0 & -.61 \\
Dublin & $' 31$ & 151.0 & 124.1 & -.78 \\
Scripps & $' 28$ & 171.5 & 120.5 & -.42 \\
Scripps & $' 31$ & 144.6 & 124.1 & -.89 \\
Scripps & $' 33$ & 146.0 & 125.7 & -.92 \\
Scripps & $' 35$ & 155.0 & 127.4 & -.80 \\
Scripps & $' 43$ & 160.5 & 136.7 & -.90 \\
Scripps & $' 47$ & 162.0 & 144.1 & -1.02 \\
\hline
\end{tabular}


$\Delta r$. The previous analysis indicates that there are at least two parts to the error: a term that depends on the year that the projection was made, and a random error term. To understand the distribution of $\Delta r$, therefore, we must study the distribution of both the jump-off error and the random error.

\section{ANALYSIS OF UNITED NATIONS PROJECTIONS}

To get a better idea about the possible size of the jumpoff error we need more data. The United Nations $(1954,1958,1966,1972)$ has made projections from jumpoff years 1950, 1955, 1960, and 1965 for target years 1955 , $1960,1965,1970$, and 1975 . They use the same component method of projection as that of the U.S. Census Bureau. They divide the world into 24 regions and make projections for each. The boundaries and number of regions change from time to time, but detailed tables allow one to put together projections for the present 24 regions. At this time we can calculate $\Delta r$ for 14 projections for each region. The results appear in Table 3 .

The first step in the analysis of these data is the calculation of the jump-off error, $b_{i j}$, for each region $i$ and jump-off year, $j$, as the mean, over all durations $k$, of $\Delta r_{i j k}$. The residual is then defined as $e_{i j k}=\Delta r_{i j k}-b_{i j}$.

Two stem-and-leaf plots (Tukey 1977) in Figure 1 show the distribution of the jump-off-error terms for the developed and developing regions, as defined by the U.N. The median jump-off error for the developed countries is +.02 , almost zero. In the long run, the U.N. projections for developed regions have been essentially unbiased, although for any given region and jump-off year, the mean of $\Delta r$ ranges from -.91 to +.40 . The median jump-off error for developing regions is -.27 and the range is -1.55 to +.56 . Over the years the U.N. has been underestimating future population and has had larger jump-off errors for developing countries.

Figure 2 breaks the data down by jump-off year. Each boxplot (Tukey 1977) schematically describes the distribution of $b_{i j}$ for each of the four jump-off years. We see that the distribution of the error for the developed countries has remained relatively stable over the four jumpoff years. In none of the years have the U.N. projections been strongly biased, and the magnitude of the error has remained approximately the same. Only in $1968 \mathrm{did}$ it tend to predict larger populations than eventually appeared. In short, as far as bias goes, the earlier projections are about as good as the later ones; the U.N.'s ability to predict does not seem to be getting either better or worse.

We see quite a different picture for the developing regions. First, the earlier projections were severely biased downward, but the later ones were less severely biased. Second, the variance of the jump-off-error term from region to region has not changed drastically over time. The jump-off term reflects errors in the basic assumptions at the time the projection is made. The increasingly better data for developing countries have allowed the U.N. de-
DEVELOPED REGIONS

\begin{tabular}{r|l}
$F$ & $(40$ \\
$T$ & $20,21,21,22,27,30,34$ \\
$0 \cdot$ & $01,03,03,04,04,05,09,10,16,16,17,18$ \\
-0. & $00,03,10,13,15,17,17$ \\
$T$ & $23,23,24,27,27,34$ \\
$F$ & 41,48 \\
$S$ & \\
$\star$ & 91
\end{tabular}

DEVELOPING REGIONS

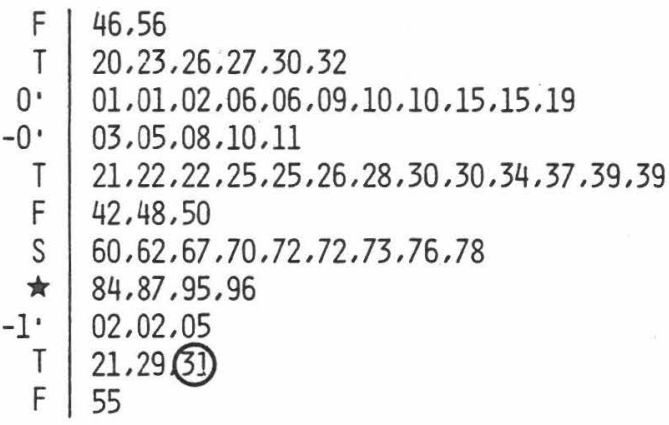

Figure 1. STEM-AND-LEAF PLOT FOR JUMP-OFF YEAR BIAS. Figures on the left of the solid line correspond to the whole number part and digits on the right relate to the first two decimal places of each $b_{11}$. Each whole number is represented by five horizontal lines: for numbers with zero or one as the first decimal digit, $T$ for two or three, $F$ for four or five, $S$ for six or seven, and "for eight or nine. For instance, the circled " 40 " means a $b_{l,}$ of +.40 for some jumpoff year in a developed region. The circled " 31 " indicates $a b_{i j}$ of -1.31 for an underdeveloped region.

mographers to reduce their jump-off error in later projection periods. An optimistic view is that future U.N. projections will have a distribution of jump-off errors centered around zero but with the same variance as each of the four years shown in Figure 2.

The stem-and-leaf plots of the residuals for developed and developing regions in Figure 3 show a larger residual variance for developing regions. Since jump-off errors have been subtracted, both distributions are centered at zero. The distribution of the error term is symmetric, and except for occasional large outliers, has a roughly normal shape. The large outliers can arise from unforeseen natural or political disasters or major errors in assumptions.

The residuals can be broken down in terms of jumpoff year and duration. Displays similar to Figure 2 indicate no change in median residual or residual variance associated either with jump-off year or with duration.

An analysis of variance of the $\Delta r$ values yields similar results. By introducing a set of dummy variables for jump- 
Table 3. $\Delta r$ for U.N. Projections

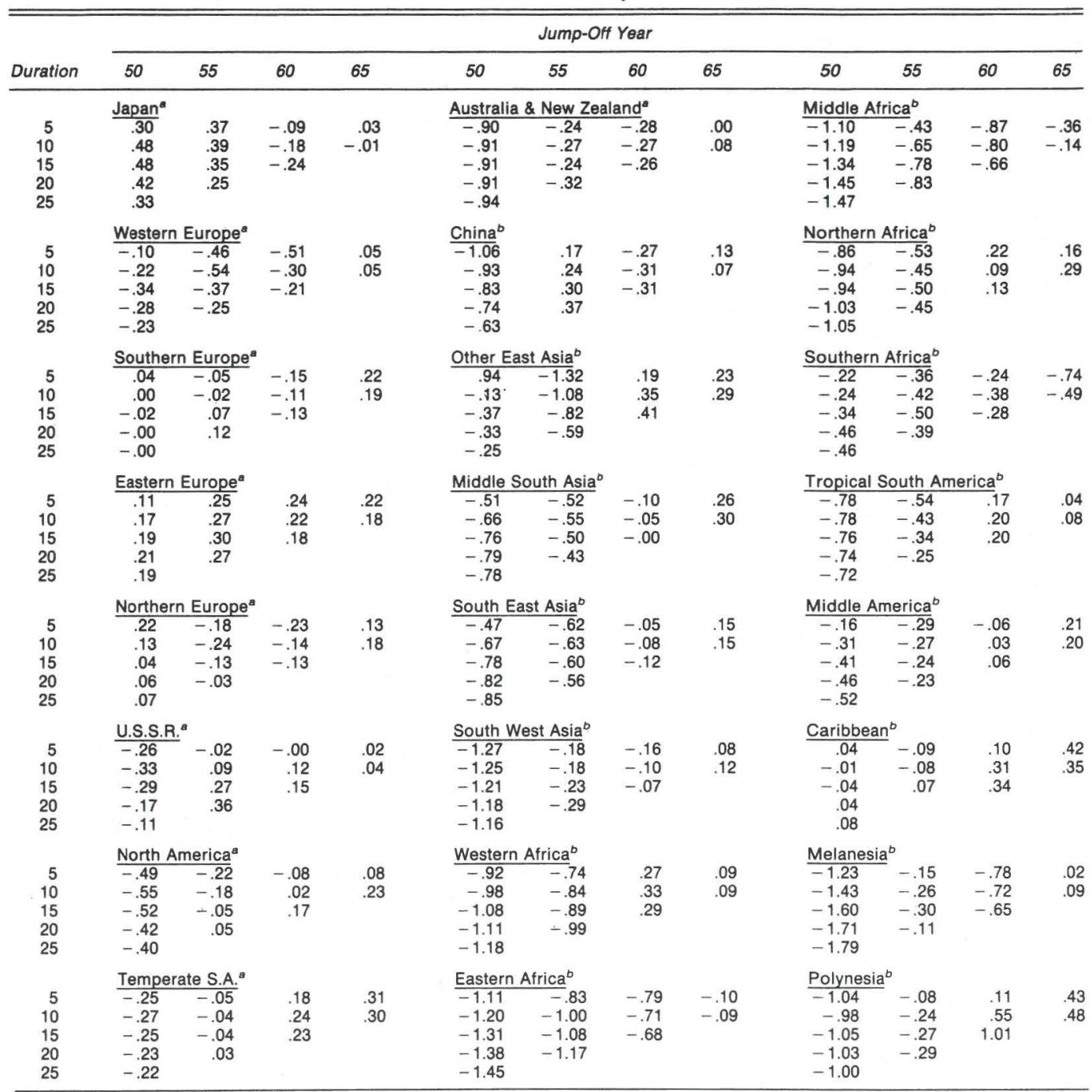

Developed region

Underdeveloped region

Source: United Nations (1954, 1956, 1966, 1972, 1977).

off year, target year, and duration into a regression analysis of $\Delta r$, we can measure the contribution of each factor to the overall variability. For developed and developing regions, we find that the jump-off year effects are significant $(F=4.49$ with 3 and 122 degrees of freedom for developed, and $F=48.05$ with 3 and 206 degrees of freedom for developing), but that, given this variable in the model, neither target year nor duration effects are significant $(F<1)$.
In summary, we break $\Delta r_{i j k}$ into two components, a jump-off-year error, $b_{i J}$, and another term, $e_{i j k}$, which seems to be a random error. For developed regions the distribution of $b_{i j}$ is stable over time and centered around zero. For developing regions, the variance of $b_{i j}$ is stable, but the distribution has been centered below zero in the past, although it is centered near zero in the latest projections. The distribution of residuals, $e_{i j}$, is stable over jump-off year and duration but has occasional outliers. 


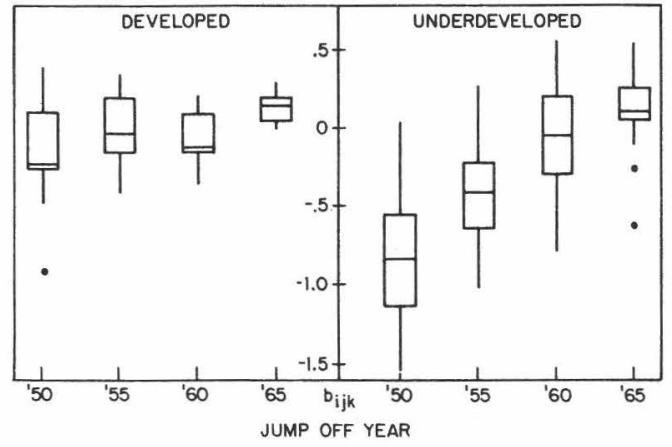

Figure 2. DISTRIBUTION OF BIAS TERM. The center horizontal line corresponds to the median of the batch of numbers, and the upper and lower limits of the box correspond to the upper and lower fourths or quartiles of the data. The box therefore represents the central half of the data. We define a point to be an outlier if it is more than $1 \frac{1}{2}$ times the length of the box from the nearest fourth. The long vertical lines connect the furthest nonoutlying point to the box, and the outliers are marked with a heavy dot.

The variance of both the jump-off error and the residual distributions is larger for developing countries.

\section{CONFIDENCE INTERVALS FOR UNITED STATES POPULATION PROJECTIONS}

We now return to the original purpose of this article, the calculation of confidence intervals for population projections. The analysis of the United States data shows that two components made up projection errors. The analysis of the U.N. data indicates that at least for the developed countries, the distribution of these terms is relatively stable. Given these conditions, we now use the observed error distributions to infer bounds on $\Delta r$, and hence $P_{T}$, for the future.

The standard deviation of the 21 values of $\Delta r$ given in Table 1 for the United States, after the jump-off-year error has been removed, is .13. The standard deviation of the jump-off-error term (based on the five observations) is .50. An estimate of the variance of $\Delta r$ is then, $\operatorname{var}\left(\Delta r_{i j k}\right)=\operatorname{var}\left(b_{i j}\right)+\operatorname{var}\left(e_{i j k}\right)=.50^{2}+.13^{2}=.27 ;$ that is, the standard deviation of $\Delta r$ is approximately .52 . In other words, the standard deviation of the predicted growth rate is about .52 percent for a population that has grown at a rate between 1 percent and 1.5 percent.

Ideally, to construct confidence intervals for U.S. Census Bureau projections, we would like to consider only United States data. But as we have seen, these data contain only 5 highly correlated observations of the jumpoff-year error, not enough to reliably estimate its variance. Instead, we use the error distributions for the U.N. developed regions. That is, lacking enough direct evidence, we consider a larger bank of data for similar regions.

The standard deviation of the jump-off term for developed regions given in Figure 3 is .27. The standard
DEVELOPED REGIONS

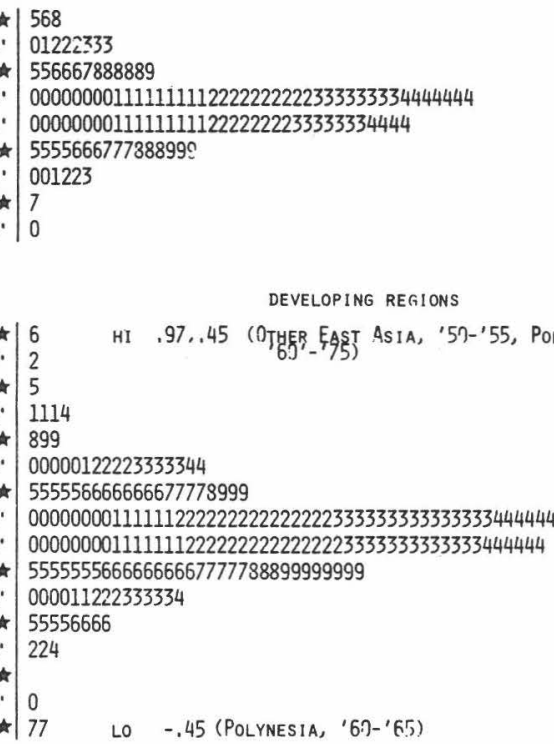

Figure 3. STEM-AND-LEAF PLOT FOR RESIDUAL ERROR. Fig ures on the left of the solid line correspond to the whole number part and digits on the right to the first decimal place. Each whole number is represented by two horizontal lines: - for numbers with 0 through 4 as the first decimal digit, and * for numbers with 5 through 9.

deviation of the error term is $\mathbf{. 0 8}$. Together these figures yield an estimated standard deviation for $\Delta r$ of .28. This estimate of standard deviation is about half of the estimate based on the U.S. Census Bureau projections, but since it is based on more independent observations, it may more accurately reflect the true variation of $\Delta r$.

The two estimates give us an order of magnitude estimate and a range of possibilities for $\sigma$, the standard deviation of $\Delta r$. We will optimistically use a value of $\sigma=$ .3 for United States projections. This means a standard deviation of .3 percent for the projected growth rate.

To construct a confidence interval, we assume that $\bar{r}_{\text {true }}$ is in the range ( $\left.\bar{p}_{\text {proj }} \pm 2 \sigma\right)$ with probability .95 , and that $\bar{r}_{\text {true }}$ is in the range $\left(\bar{r}_{\text {proj }} \pm \sigma\right)$ with probability $\frac{2}{3}$. These values would be approximately true if $\Delta r$ had a Normal distribution and are good approximations in other cases, especially given the nearly Normal shape of the distribution in Figures 1 and 3.

Using the relationship

$$
P_{T}=P_{0} \exp \left(T\left(\bar{r}_{\text {true }}\right)\right),
$$

a 95 percent confidence interval for $\boldsymbol{P}_{T}$ is approximately $\left(P_{0} \exp \left(T\left(\bar{r}_{\text {proj }}-2 \sigma\right), P_{0} \exp \left(T\left(\bar{r}_{\text {proj }}+2 \sigma\right)\right)\right.\right.$ and a $\frac{2}{3}$ interval $\left(P_{0} \exp \left(T\left(\bar{r}_{\text {proj }}-\sigma\right), P_{0} \exp \left(T\left(\bar{r}_{\text {proj }}+\sigma\right)\right)\right.\right.$. Figure 4 plots these intervals for the optimistic estimate $\sigma=.3$. For the turn of the century the $\frac{2}{3}$ interval is 241 to 280 million 


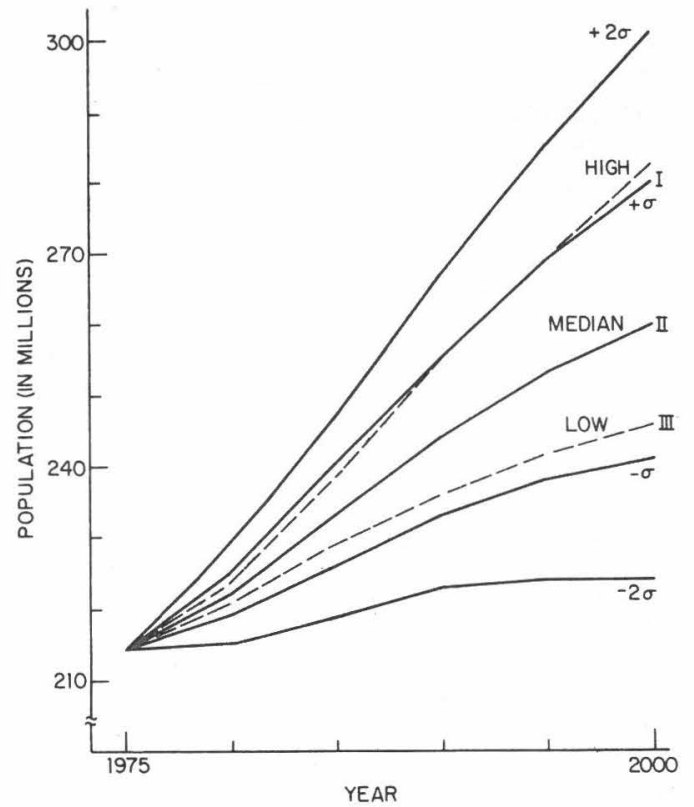

Figure 4. U.S. CENSUS PROJECTIONS (HIGH, MEDIAN, LOW $A N D \sigma=.3$ CONFIDENCE INTERVALS

and the 95 percent interval is 224 to 302 million. For comparison, the Census Bureau's (1977) most recent high and low projections are also shown in Figure 4. They correspond approximately to the $\frac{2}{3}$ interval. A more pessimistic analysis, with $\sigma=.5$ based solely on United States data, would give confidence intervals approximately twice as wide.

\section{OTHER METHODS FOR PROJECTING POPULATIONS}

So far we have examined two similar sets of projections-both made by the component method for largescale regions. To gain some perspective we examine in this section two other types of population projection.

Long (1977) presents four sets of population projections for the 50 .American states from 1970 to 1975 . Two are standard demographic projections made by the U.S. Census Bureau and the National Planning Association. A third is similar to the Census Bureau's projection but assumes no internal migration. The fourth projection, by the U.S. Bureau of Economic Analysis, is based on economic rather than demographic assumptions. The mean value and standard deviation of $\Delta r$ for each of these sets of projections appear in Table 4. Each set is much more variable than the United States or U.N. projections, and they are all, especially the economic projections, seriously biased.

A simple and common technique for projecting populations is to assume that the growth rate during the next
$T$ years will be the same as it was during the last $T$ years. This assumption yields the geometric projection formula

$$
\hat{\boldsymbol{P}}_{T}=\frac{\boldsymbol{P}_{0}}{\boldsymbol{P}_{-T}} \cdot \boldsymbol{P}_{0}
$$

The U.N. data allow us to evaluate this technique four times for each of the 24 regions for $T=5$ and two times for each region for $T=10$. The mean and standard deviations of the $\Delta r$ are given in Table 3. For these data, the simple geometric projection technique has been almost unbiased and has a standard deviation equal to or smaller than the more complex methods.

These results indicate that, for some purposes, the simplest projection method is better than the more complicated models. Certainly its simplicity and the small amount of data necessary for its application speak in its favor. On the other hand, it has not been adequately tested for durations longer than 10 years. Furthermore, the geometric method only predicts total population size, not age composition, as does the component method. Sometimes, for instance when we are planning for the educational or Social Security system, this age composition is exactly what we need. So for short-term, total population projections simple geometric projection gives more accurate results than the more complicated component method.

\section{COMPARISON OF PROJECTION TECHNIQUES}

Table 4 sums up the evidence we have gathered in this article about population projections. Population projections for countries or regions tend to have a standard deviation of about .3 or .5 in $\Delta r$, which means an error of \pm .3 or \pm .5 percent per year in growth rates that range from .5 to 2.5 percent per year. Errors for developed regions are smaller than for developing regions. Subnational projections are one-half to one-third as accurate (in terms of standard deviation) as national or regional projections and are biased as well. Simple geometric projections have been relatively unbiased and accurate for total population size. This last fact gives extra support for the choice of the definition of $\Delta r$.

Table 4. Summary of Projection Errors

\begin{tabular}{lccc}
\hline \hline \multicolumn{1}{c}{ Projection Series } & Bias & $\begin{array}{c}\text { Standard } \\
\text { Deviation }\end{array}$ & $\begin{array}{c}\text { Sample } \\
\text { Size }\end{array}$ \\
\hline U.S. Census Bureau & -.02 & $.50^{\mathrm{a}}$ & 21 \\
U.N. Developed Regions & -.03 & $.28^{\mathrm{a}}$ & 126 \\
U.N. Underdeveloped & -.34 & $.51^{\mathrm{a}}$ & 210 \\
Regions & -.23 & $.92^{\mathrm{a}}$ & 50 \\
$\quad$ Census I-E & -.09 & $.76^{\mathrm{b}}$ & 50 \\
$\quad$ National Planning Assoc. & -.41 & $.93^{\mathrm{b}}$ & 50 \\
$\quad$ U.S. Bureau of Economic & & & \\
$\quad$ Analysis & -.16 & $.95^{\mathrm{b}}$ & 50 \\
$\quad$ Census III-E & & & \\
Constant Geometric Growth & -.005 & $.19^{\mathrm{b}}$ & 96 \\
$\quad \mathbf{5}$ years & -.01 & $.32^{\mathrm{b}}$ & 48 \\
\hline 10 years & & & \\
\hline
\end{tabular}

- Based on separate analysis of bias and error terms.

- Sample standard deviation. 


\section{LIMITATIONS OF THIS APPROACH}

Three limitations of the data-analytical approach of this article follow. First, we treat all of the $\Delta r$ as independent random observations, the actual population sizes from year to year are not independent, and all projections made at one time depend on a common set of assumptions. This error is not serious when one considers the error between two fixed points in time, but from our analysis it is impossible to make simultaneous confidence intervals for two or more future populations. Were longer data series available, formal methods of time series analysis could help us understand these temporal correlations.

Second, $\Delta r$ reflects only the error in total population size, and not the error in age composition. Sometimes future age composition, not size, is the main goal of population prediction. But more frequently, the total population size is the more important quantity, and the $\Delta r$ analysis allows us to construct confidence intervals for it. The same argument applies to geographic and other stratifications.

Third, sometimes the aim of a population projection is not for predictive purposes but is to provide a warning about the consequences of present trends. One could argue that these projections are successful only if they are wrong. We do not regard $\Delta r$ as a measure of success, but simply as a measure of the difference between actual and projected populations. It is a measure of the accuracy of projections if, as is commonly the case, they are interpreted as predictions.

\section{CONCLUSIONS}

A historical analysis of certain series of population projections shows that

1. the yearly growth rate error, $\Delta r$, allows a simple and coherent picture of the error structure of population projections;

2. this error, $\Delta r$, consists of two factors, a term as sociated with the jump-off year and a random error term;

3 . the distribution of both factors has been relatively stable over time.

The discovery of stable error distributions allows us to transform the results of the historical analysis into confidence intervals for future populations. These confidence intervals reflect the best efforts of competent demographers in the past and should be a reliable guide to the present generation's ability to predict the future.

The resulting confidence intervals for the United States are very large. An optimistic analysis gives a $\frac{2}{3}$ confidence interval approximately equal to the Census Bureau's low and high estimates. A 95 percent interval for the year 2000 ranges from about 220 to 300 million. State populations are harder to predict accurately. Simple geometric projections of total population for short durations are slightly more accurate.

Population projections are important tools for planning and policy analyses. But they can never exactly foretell the future. We do not intend to criticize the construction or use of population projections, nor do we pretend to be able to improve them. Instead we merely attempt to measure their inherent inaccuracy. This analysis enables planners to use projections more objectively by providing a range of reasonable possibilities rather than a single estimate.

\section{EPILOGUE}

Since the original presentation of these results, the 1980 U.S. Census figures have become available, thus offering one comparison of the true population with the proposed confidence interval. According to the latest Census Bureau (1977) projection, the Series I, II, and III estimates for 1980 are respectively, 224.1, 222.2, and 220.7 million. The $2 \sigma$ confidence interval in Figure 4 is 229.0 to 213.3 million. The actual census count is 226.5 million, higher than the Series I projection but within the $2 \sigma$ interval.

[Received October 1981. Revised July 1982.]

\section{REFERENCES}

COHEN, J.E. (1976), "Ergodicity of Age Structure in Populations With Markovian Vital Rates. I: Countable States," Journal of the American Statistical Association, 71, 335-339.

(1977a), "Ergodicity of Age Structure in Populations With Markovian Vital Rates. II: General States," Advanc's in Applied Prob-

ability, 9, 18-37. kovian Vital Rates. III: Finite-State Moments and Growth Rates; Illustrations," Advances in Applied Probability, 9, 462-475.

DORN, H.F. (1950), "Pitfalls in Population Forecasts and Projections," Journal of the American Statistical Association, 45, 311-334.

ISSERMAN, A.M. (1977), "The Accuracy of Population Projections for Subcountry Areas," Journal of the American Institute of Planners, 43, 247-259.

KEYFITZ, N. (1972), "On Future Populations," Journal of the American Statistical Association, 67, 347-363.

LEE, R.D. (1974), "Forecasting Births in Post-Transition Populations: Stochastic Renewal With Serially Correlated Fertility," Journal of the American Statistical Association, 69, 607-617.

LONG, J.F. (1977), "Prospects for a Composite Demographic-Economic Model of Migration for Subnational Projections," in Report of the Conference on Economic and Demographic Methods for Projecting Population, Washington, D.C.: American Statistical Association.

PITTENGER, D.B. (1978), "The Role of Judgment, Assumptions, Techniques and Confidence Limits in Forecasting Population," Socio-Economic Planning Science, 12, 271-276.

SABOIA (1974), "Modeling and Forecasting Populations by Time Series: The Swedish Case," Demography 11, 483-492.

(1977), "Auto-Regressive Integrated Moving Average (ARIMA) Models for Birth Forecasting," Journal of the American Statistical Association, 72, 264-270.

SIEGEL, J.S. (1972), "Development and Accuracy of Projections of Population and Households in the United States," Demography, 9, $51-68$

SYKES, Z.M. (1969), "Some Stochastic Versions of the Matrix Model for Population Dynamics," Journal of the American Statistical Association, 44, 111-130

TUKEY, J.W. (1977), Exploratory Data Analysis, Reading, Mass.: Addison-Wesley.

UNITED NATIONS (1954), "Framework for Future Population Estimates, 1950-1980, by World Regions," Proceedings of the World Population Conference, 1954, 3, 283-328.

(1958), The Future Growth of World Population, Population Studies 28, ST/SOA/Series A/28, New York.

(1966), World Population Prospects as Assessed in 1963, Population Studies 41, ST/SOA/Series A/41, New York. 
(1972), World Population Prospects, 1965-2000, as Assessed in 1968, Population Studies 53, ST/SOA/Series A/53, New York.

(1977), World Population Prospects as Assessed in 1973, Population Studies 60, ST/ESA/Series A/60, New York.

U.S. BUREAU OF THE CENSUS (September 1946), Population Special Reports, Series P-46, No. 7.

(August 1953), Current Population Reports, Series P-25, No. 78

-(November 1958), Current Population Reports, Series P-25, No.
- (July 1962), Current Population Reports, Series P-25, No. 251. (March 1966), Current Population Reports, Series P-25, No. 329. (November 1971), Current Population Reports, Series P-25, No. 470. WHITE, H.R. (1954), "Empirical Study of the Accuracy of Selected Methods of Projecting State Populations," Journal of the American Statistical Association, 29, 480-498. 